\title{
Einige grundlegende Fragen der Modernisierung des ungarischen Hochschulwesens
}

\author{
Dr. László Dinya
}

\section{Die Herausforderungen des ungarischen Hochschulwesens}

Die Wandlungen im ungarischen Hochschulwesen kann man nur verstehen, wenn sie in sozialen und wirtschaftlichen Zusammenhängen analysiert werden. Jeglicher Versuch, diese Veränderungen ausschließlich auf innere (innerhalb des Hochschulwesens definierte) Ursachen zurïckzuführen, ist zwangsläufig zum Scheitern verurteilt.

Die Frage ist: Welchen Weg wird das Hochschulwesen gehen? Oder anders gefragt, welchen Weg muß es unter Berüicksichtigung der gegenwärtig wirkenden Sachzwänge gehen? Die wichtigsten gegenwärtig besonders spürbaren Sachzwänge sind folgende:

Ein kurzfristig wirkender Sachzwang ergibt sich aus der bevorstehenden Reform des Staatshaushaltes. Die Rolle des Staatsetats hat sich innerhalb kurzer Zeit wesentlich verändert. Bei finanziellen Zuwendungen werden vorrangig Gesichtspunkte der Effektivität berüicksichtigt; dabei ist mit wesentlich geringeren staatlichen Finanzierungen zu rechnen als bisher. Dadurch gelangt das Hochschulwesen in eine ganz neue, von der früheren wesentlich abweichende Situation. Die Bedürfnisse des Hochschulwesens können nicht mehr in dem Maße berïcksichtigt werden wie früher. Die Finanzierung der Einrichtungen wird auf eine neue Basis gestellt. Dies schließt eine soziale Neustrukturierung des Studiensystems ein (einerseits leistungsorientierte Finanzierungen auf verschiedene Weise durch Stipendien, soziale Unterstützung sowie andererseits die Zahlung von Studiengebühren müssen berücksichtigt werden, wobei das gesamte System auch Kompensationsmöglichkeiten enthalten sollte). All das führt zur Neubestimmung der Aufgaben der Institutsleitungen, die eine stärkere persönliche Haftung im Sinne der Unternehmensführung im Gegensatz zur staatlichen Lenkung beinhaltet. Die institutionelle Autonomie muß im Hinblick auf die fachliche Autonomie der Einrichtungen neu definiert werden und die kontrollierte Verwendung der öffentlichen Gelder garantieren. Da sich das Hochschulwesen von seinem Systemcharakter her nur langsam verändert, müssen die Folgen der Staatshaushaltsreform rechtzeitig von denjenigen erkannt werden, die für das Management und die Leitung der Institutionen zuständig sind. Die Reform des Staatshaushaltes wird innerhalb kurzer Zeit kommen und die Institutionen, die sich in ihrer Struktur auf die damit verbundenen Veränderungen nicht vorbereiten, können sehr schnell an den Rand der Funktionsfähigkeit geraten.
Ein längerfristig wirkender Sachzwang für das ungarische Hochschulwesen besteht darin, die Direktiven des Parlamentsbeschlusses über die Entwicklungsaufgaben des Hochschulwesens umzusetzen. Die fünfzehn im Jahre 1995 verabschiedeten Direktiven definieren die Aufgaben für die europäische Integration des ungarischen Hochschulwesens. Die Mehrheit dieser Aufgaben ist natïrlich nur langfristig realisierbar. Manche Experten betonen zu Recht, daß unsere Ergebnisse schon auf europäischem Niveau wären und daher die Modernisierung des Hochschulwesens nur noch eine scheinbare Aufgabe sei. Das ist zwar richtig, aber wir erzielen diese Ergebnisse bislang mit osteuropäischen Methoden (Institutsmanagement), die in dieser Form nicht mehr aufrecht erhalten werden können. Die Anzahl der Studierenden muß erhöht, neue Bildungsformen müssen eingeführt, die Stundenzahl muß reduziert, Qualitätsgarantien müssen in den Instituten ausgebaut und ein entsprechendes Vergütungssystem muß eingeführt werden. All das sind Aufgaben, die trotz vorhandener Anfangserfolge erst nach längerer Zeit zu den gewünschten Resultaten fuihren. Natürlich bleibt die Zuständigkeit der Regierung für rechtliche Regelungen erhalten, aber in vielen Fällen gibt es bereits unter den gegenwärtigen Bedingungen vielfältige Möglichkeiten für Initiativen der Institutionen selbst (Mikroebene). Die rechtzeitige Analyse der Sachzwänge ermöglicht es, mit der Ausarbeitung der Strategien auf Institutionsebene bereits zu beginnen, denn ohne diese ist ein durchdachtes Management kaum vorstellbar.

Ein ständiger Sachzwang für das Hochschulwesen resultiert aus den sich fortwährend wandelnden (aber berechenbaren) Herausforderungen des Arbeitsmarktes. Die heutige Zulassungspolitik der Hochschulinstitutionen in Ungarn berücksichtigt den Arbeitsmarkt und die sich vollziehenden Veränderungen kaum. Als gegenwärtige Entwicklung zeichnet sich dabei ab, daß die gesellschaftliche Nachfrage nach Hochschulangeboten auf der Input-Seite wegen der demographischen Welle, der Ängste vor der Arbeitslosigkeit und des steigenden Prestiges/Images der Bildung an sich steigt. Die Anzahl der Studierenden hat sich in den Jahren 1990 bis 1997 um $50 \%$ erhöht. Die Verteilung der Studierenden auf die verschiedenen Hochschultypen hat sich hingegen in den letzten 20 - 30 Jahren kaum verändert. Die Zulassungspolitik ist im Großen und Ganzen die gleiche, und die Zunahme der Anzahl der Studierenden erfolgt ausschließlich extensiv (die Struktur ändert sich nur wenig). Aus diesem Grund und natülich auch als Resultat der Inflation werden immer mehr Studierende mit immer mehr Kosten ausgebildet. Um einen Arbeitsplatz zu fin- 
den, müssen sie nach dem Abschluß des Studiums zusätzlich investieren und in weiteren Studienabschnitten zusätzliche Qualifikationen erwerben. Es ist leider eine Tatsache, daß in den Hochschulinstitutionen von heute ein neuer Studiengang oder Schwerpunkt in vielen Fällen wegen überkommener interner Konflikte nicht oder nur unter schwierigen Bedingungen eingefuihrt werden kann. Noch schwieriger ist es (manchmal unmöglich), die Institutionen zu veranlassen, mit einem veralteten Programm aufzuhören. Seit 1997 wird unter Berücksichtigung der staatlich finanzierten Studentenanzahl eine Aufnahmebegrenzung herausgegeben, die von Arbeitsmarkttendenzen ausgeht und als unentbehrlicher Bestandteil der normativen Finanzierung gilt. Das bedeutet $z$. B. für eine Institution, die sich auf einem ziemlich schmalen Gebiet profiliert hat und nur wenig nachgefragte Studiengänge anbietet, daß sie in Folge der normativen Finanzierung in Bedrängnis geraten kann. Sie muß sich auf limitierte Aufnahmequoten vorbereiten. Es gibt noch weitere Sachzwänge, wie z. B. die negative demographische Entwicklung, die sich im ständigen Wandel befindliche Volkswirtschaft, die Integration Ungarns in die EU und als deren Folge die Öffnung des europäischen Arbeitsmarktes oder der sogenannte „Brain-drain“ talentierter Lehrkräfte. Das sind Tendenzen, die das Hochschulwesen einfach nicht außer acht lassen darf.

\section{Die Grundprinzipien der Modernisierung des Hochschulwesens}

Die Modernisierung des Hochschulwesens ist keine Kampagne. Sie ist eine ständige langfristige Aufgabe (ein Prozeß), der sich nach folgenden Grundprinzipien vollzieht:

Das Hochschulwesen befindet sich in ständiger Wechselwirkung mit der Gesellschaft und der Wirtschaft. Es muß die neuen Bedürfnisse des Wandels nicht nur wahrnehmen, sondern die vorrangige Aufgabe besteht darin, sie aufzudecken, darauf zu reagieren, an ihrer Gestaltung mitzuwirken und die Hochschulangehörigen für die Lösung dieser Aufgaben zu motivieren. Die größten Herausforderungen für das Hochschulwesen sind zur Zeit folgende:

- Anpassung an die langfristig prognostizierbaren Ansprüiche des Arbeitsmarktes (Zulassung auf Grund des Output, neue Bildungsprogramme),

- Erreichung einer in entwickelten Ländern üblichen Zulassungszahl ( $25-30 \%$ der Altersgruppe zwischen 18 und 24 Jahren),

- Aufrechterhaltung renommierter ungarischer Hochschulen (Schulen, Theorien, weltbekannte Institutionen),

- Zurüickdrängung negativer Tendenzen (Problem des Hochschullehrernachwuchses, der Gegenselektion, des praxisbezogenen Unterrichts),

- Beseitigung kontraproduktiver Tendenzen zur Modernisierung (Veränderung der Institutionsstruktur, des Institutions- und Wirtschaftsmanagements und des Niveaus der Infrastruktur).
Alle diese Aufgaben laufen parallel mit der Reform des Staatshaushaltes. Sie sind teils eigenständige Aufgabenbereiche und teils eng damit verbunden.

Die Reaktion auf diese Herausforderungen bedarf einer komplizierten, abgestimmten und langfristig angelegten Reihe von Maßnahmen. Kurzfristig sind dabei folgende Teilaufgaben zu lösen:

- Modifizierung des Hochschulgesetzes,

- Institutionsintegration,

- Einfuihrung der normativen Finanzierung,

- Korrektur des Systems der sozialen Unterstuitzung der Studierenden.

Die langfristigen Aufgaben sind in den Direktiven des Parlamentsbeschlusses über die Entwicklung des Hochschulgesetzes festgelegt.

\section{Die Modifizierung des Hochschulgesetzes}

Die Modifizierung des Hochschulgesetzes LXXX. von 1993 bildet den juristischen Rahmen für die kurz- und längerfristigen Aufgaben bei der Modernisierung des Hochschulwesens. Die Modifizierung des Gesetzes beinhaltet folgende wichtige Bereiche:

- die Umgestaltung der institutionellen Struktur (die Definition der Universitäts- bzw. Hochschulintegration, Hochschulen mit mehreren Fakultäten),

- eine neue Bildungsstruktur (post-secondary Bildung, das Creditpointsystem, die Akkreditierung, das Aufnahmesystem, der Anschluß an das Output-Unterrichtssystems mittleren Grades, die Universitätsbildung auf zwei Ebenen, eine einheitliche Lehrerausbildung),

- die Modifizierung der Rolle des Staates im Hochschulwesen (die Bestimmung der Aufgaben des Kultusministeriums, des Wissenschaftlichen Rates für Hochschulwesen, des Ungarischen Akkreditierungskomitees und des Mechanismus der Interessenabstimmung),

- die institutionelle Funktion, den selbständigen Haushalt, die juristischen und organisatorischen Fragen der Autonomie.

Die Ergebnisse in diesen Bereichen können zur Lösung solcher Probleme, wie juristischer Unklarheiten bei der Integration, beitragen. Sie ermöglichen die Verwirklichung der Direktiven des Parlamentsbeschlusses. Der auf dieser Grundlage gebildete Finanzierungsrat erscheint als Körperschaft, die die Entscheidungen des wissenschaftlichen Rates für das Hochschulwesen vorbereitet.

\section{Die Institutsintegration}

Die Umgruppierung der knappen Mittel und ihre wirksamste Anwendung muß der harmonischen Entwicklung der Elemente im System dienen:

- die Entwicklung der Inhalte und des Niveaus im Hochschulwesen kann am wirksamsten durch die Integration der Institutionen gelöst werden, 
- die erwünschte Zunahme der Anzahl der Studierenden kann durch die bessere Ausschöpfung der Kapazitäten in den integrierten Institutionen verwirklicht werden.

Unabhängig davon müssen die Institutionen selbst, die Regierung und die fachlichen Körperschaften ihre Aufgaben wahrnehmen.

Die oben genannten Faktoren brauchen eine zusätzliche Finanzierung, die durch die Mobilisierung innerer Reserven erschlossen werden kann. Durch die Integration werden als Folge der Strukturreform momentan beanspruchte finanzielle Quellen freigesetzt und können zur Verfügung gestellt werden. Diese Art der Finanzierung kommt in entscheidendem Maße durch die freigesetzten Beträge des Etats zustande und kann mit neuen langfristigen Weltbankkrediten kombiniert werden. Sie dient aber ausdrücklich den Zielsetzungen der Integration. Die Integration kann also - in der gegenwärtigen Phase der Umwandlung des Hochschulwesens - als motivierende Kraft betrachtet werden.

Mögliche Formen der Integration sind:

- lockere Vereinigungen ohne Hochschulstatus. Dieses System existiert schon, aber damit wollen wir uns nicht beschäftigen,

- Universitäts-(Hochschul-)bündnisse, die in der Gesetzmodifizierung definiert werden,

- integrierte Universitäten mit mehreren Fakultäten, deren Status im Gesetz geregelt ist.

\section{Die normative Finanzierung}

Mit der Einführung der normativen Finanzierung der Hochschulinstitutionen in Abhängigkeit von der Studentenanzahl wurde 1996/97 begonnen. Damit sind eng verbunden:

- die Bestimmung der Aufnahmequote in den Fachbereichen aufgrund von Arbeitsmarktanalysen (das erste Mal für das Aufnahmeverfahren 1997 gültig),

- die Verteilung der aufgenommenen Anzahl von Studierenden aufgrund bestimmter Prioritäten (wie z. B. die der integrierten Hochschulinstitutionen).

Zugleich muß eine Datenbank zu den Anforderungen des Arbeitsmarktes eingerichtet und deren Betreuung und Pflege gewährleistet werden. Das gleiche bezieht sich auf die Angaben zur Instandhaltung der Institutionsgebäude, die die normative Finanzierung in diesem Bereich ermöglichen könnte.

\section{Harmonie mit der Staatshaushaltsreform}

Im Hinblick auf die Erfahrungen der gegenwärtigen Phase der Staatshaushaltsreform kann festgestellt werden, daß die Direktiven und die geplanten Maßnahmen zur Umwandlung des Hochschulwesens mit dem Geist der Staatshaushaltsreform bezüglich des Hochschulwesens im völligen Einklang stehen. Es ist wichtig, die- sen Sachverhalt hervorzuheben, denn das Hochschulwesen kam nach langen Diskussionen und meist fachlich motivierten Entscheidungen zu den entsprechenden Konsequenzen.

Die Effektivität ist dabei nur Mittel und nicht das Ziel der Bestrebungen nach Modernisierung, d. h. die Prioritäten der Modernisierung sind die folgenden:

(a) Es ist zu bestimmen, über welche Struktur und Funktionsregeln das Hochschulwesen verfuigen muß, um der Europäischen Union „beitreten“ zu können.

(b) Im Zentrum der gesetzten Ziele muß der Gedanke der Effektivität stehen.

In umgekehrter Reihenfolge kann die Effektivität zum Selbstzweck werden. Losgelöst von den erwünschten Ergebnissen führt sie in die Sackgasse.

\section{Verfasser}

\section{Dr. László Dinya}

Stellvertretender Staastssekretär im Kultusministerium Ungarn

H-1055 Budapest

Szalay u. 10-14

Tel. +361 - 3310795 